\title{
Performance studies of a full-length prototype for the CASTOR forward calorimeter at the CMS experiment
}

\author{
V. Andreev ${ }^{1}$, X. Aslanoglou ${ }^{2}$, A. Azman ${ }^{3}$, M.N. Bakirci ${ }^{3}$, S. Başeğmez ${ }^{3}$, W. Beaumont ${ }^{4}$, J. Blocki ${ }^{5}$, K. Borras ${ }^{6}$, \\ A. Campbell ${ }^{6}$, S. Çerçi ${ }^{3}$, D. d'Enterria ${ }^{7}$, M. de Silva ${ }^{7}$, I. Dumanoğlu ${ }^{3}$, S. Erturk ${ }^{3}$, E. Eskut ${ }^{3}$, Y. Ershov ${ }^{8}$, \\ P. Göttlicher ${ }^{6}$, L. Gouskos ${ }^{9}$, G. Onengut ${ }^{3}$, Y. Gusev ${ }^{10}$, H. Jung ${ }^{6}$, I. Katkov ${ }^{6,14}$, P. Katsas ${ }^{9}$, a , L. Khein ${ }^{6,14}$, F. Kisoglu ${ }^{3}$, \\ A. Knutsson ${ }^{6}$, S. Kuleshov ${ }^{11}$, A. Kuznetsov ${ }^{8}$, M. Lebeau ${ }^{9}$, T. McCauley ${ }^{12}$, C. Muhl ${ }^{6}$, Y. Musienko ${ }^{12,13}$, S. Ochesanu ${ }^{4}$, \\ M. Oroku ${ }^{7}$, K. Ozdemir ${ }^{3}$, S. Ozturk ${ }^{3}$, A.D. Panagiotou ${ }^{9}$, S. Reucroft ${ }^{12}$, M. Ripert ${ }^{4}$, K. Shileev ${ }^{13}$, K. Sogut $^{3,15}$, \\ J. Swain ${ }^{12}$, V. Tiflov ${ }^{13}$, A. Kayis-Topaksu ${ }^{3}$, H. Van Haevermaet ${ }^{4}$, P. Van Mechelen ${ }^{4}$, E. de Wolf ${ }^{4}$ \\ ${ }^{1}$ P.N. Lebedev Physical Institute, Russian Academy of Sciences, Moscow, Russia \\ ${ }^{2}$ Dept. of Phys., University of Ioannina, PO Box 1186, 45110 Ioannina, Greece \\ ${ }^{3}$ Dept. of Phys., University of Cukurova, 01330 Adana, Turkey \\ ${ }^{4}$ Dept. of Phys., Antwerp University, Groenenborgerlaan 171, 2020 Antwerpen, Belgium \\ ${ }^{5}$ Institute of Nuclear Physics, Radzikowskiego 152, 31342 Kraków, Poland \\ ${ }^{6}$ DESY, Hamburg, Germany \\ ${ }^{7}$ PH Dept., CERN, 1211 Geneva 23, Switzerland \\ ${ }^{8}$ Joint Institute for Nuclear Research, Dubna, Russia \\ ${ }^{9}$ Phys. Dept., University of Athens, 15701 Athens, Greece \\ ${ }^{10}$ Institute for High Energy Physics, Moscow, Russia \\ ${ }^{11}$ ITEP Institute for Theoretical and Experimental Physics, Moscow, Russia \\ ${ }^{12}$ Dept. of Physics, Northeastern University, Boston, MA 02115, USA \\ ${ }^{13}$ Institute for Nuclear Research, Moscow, Russia \\ ${ }^{14}$ Moscow State University, Moscow, Russia \\ ${ }^{15}$ Mersin University, Mersin, Turkey
}

Received: 5 November 2009 / Revised: 19 March 2010 / Published online: 30 April 2010

(C) The Author(s) 2010. This article is published with open access at Springerlink.com

\begin{abstract}
We present performance studies of a full-length prototype for the CASTOR quartz-tungsten sampling calorimeter, installed in the very forward region of the CMS experiment at the LHC. The response linearity and energy resolution, the uniformity, as well as the showers' spatial properties in the prototype have been studied with electrons, pions and muons of various energies. A special study was also carried out for testing the light-output with a 90-degree cut of the quartz plates of the calorimeter. The data were taken during the CASTOR test beam at CERN/SPS in 2007.
\end{abstract}

\section{Introduction}

The CASTOR (Centauro And Strange Object Research) detector is a Čerenkov quartz-tungsten sampling calorimeter, which is currently installed in the CMS experiment at $14.4 \mathrm{~m}$ from the interaction point, covering the pseudorapidity range $-5.2<\eta<-6.6$ [1]. The detector will contribute mainly to forward QCD studies (diffractive, low- $x$ )

a e-mail: Panagiotis.Katsas@ cern.ch and cosmic-rays-related physics in both proton-proton [2] and heavy-ion [3] collisions at LHC energies. A detailed description of the principle of functioning has been provided in previous references $[1,4,5]$. The calorimeter is constructed from layers of tungsten alloy $\left(\mathrm{W}: \lambda_{I}=10.0 \mathrm{~cm}\right.$, $X_{0}=0.365 \mathrm{~cm}$, density $=18.5 \mathrm{~g} / \mathrm{cm}^{3}$ ) plates as absorber and silica quartz (Q) plates as active medium (Fig. 1). For the electromagnetic section, the W-plates have a thickness of $5 \mathrm{~mm}$ and the Q-plates $2 \mathrm{~mm}$. For the hadronic section, the $\mathrm{W}$ - and Q-plates have a thicknesses of $10 \mathrm{~mm}$ and $4 \mathrm{~mm}$, respectively. The effective thickness of the plates is larger, as they are inclined $45^{\circ}$ with respect to the direction of the impinging particles, in order to maximize the Čerenkov light output in the quartz. ${ }^{1}$ Each pair of W/Q-plates is defined as a sampling unit (SU). Overall, the calorimeter is segmented azimuthally in 16 semi-octants (sectors) and longitudinally

\footnotetext{
${ }^{1}$ The refractive index of quartz is $n=1.46-1.55$ (for wavelengths $\lambda=600-200 \mathrm{~nm})$. The corresponding Čerenkov threshold velocity and angle are $\beta_{c}=1 / n=0.65-0.69$ and $\theta_{c}=a \cos (1 / n \beta)=46^{\circ}-50^{\circ}$ respectively.
} 
Fig. 1 CASTOR tungsten/silica quartz plates (left) and photomultipliers on top of the light guides (right) as installed on the prototype calorimeter that was beam tested in 2007 at SPS
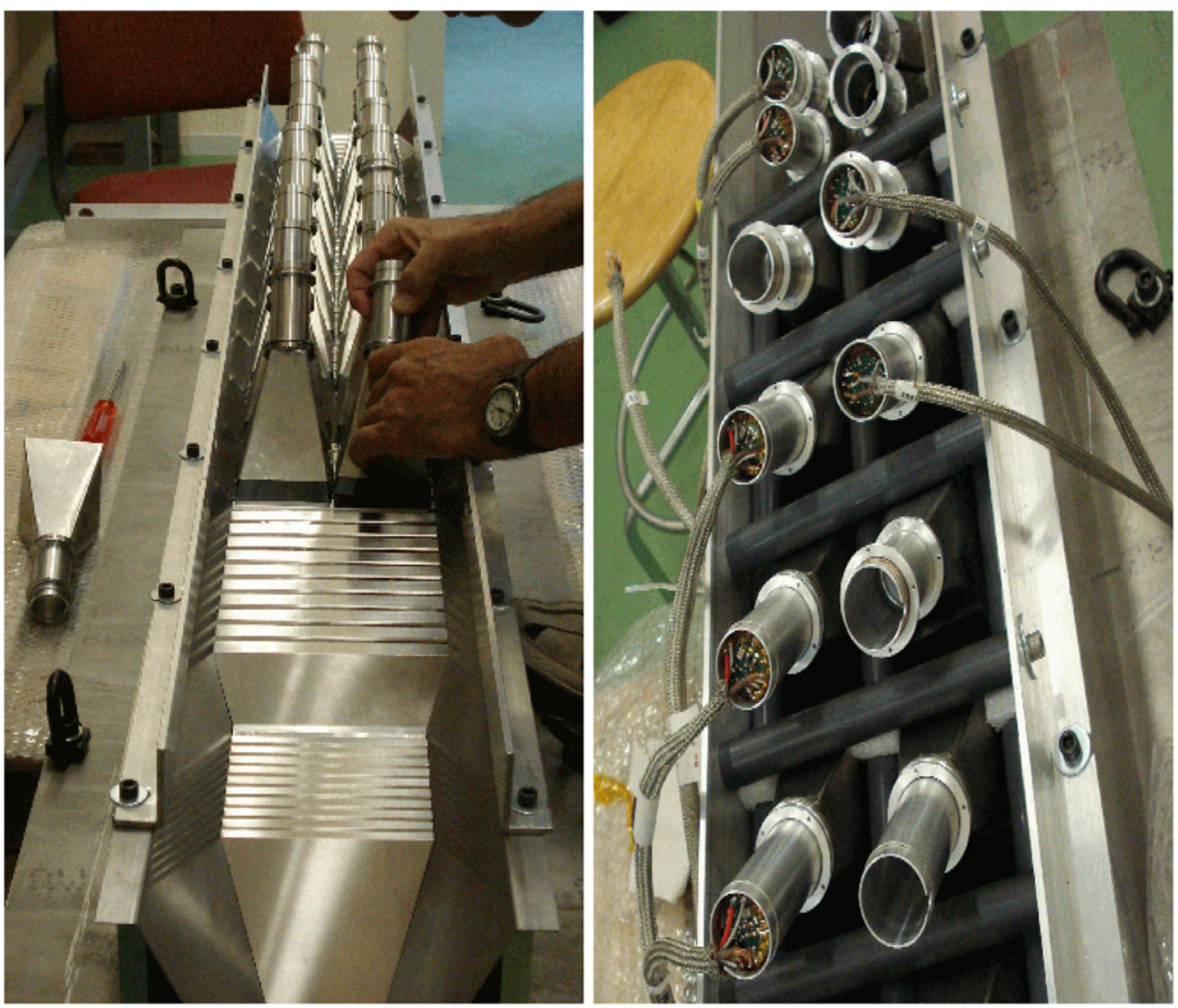

in 14 channels per sector. The electromagnetic section is divided in two successive channels, each one consisting of 5 sampling units, and is $10.06 X_{0}$, or $0.385 \lambda_{I}$ deep. In total, the EM section corresponds to $20.12 X_{0}$ or $0.77 \lambda_{I}$ lengths. In the hadronic section, each channel consists also of 5 sampling units of $0.77 \lambda_{I}$ depth each. In total there are 12 hadronic channels, corresponding to $9.24 \lambda_{I}$. The entire CASTOR prototype is approximately 10 interaction lengths. The Čerenkov light emitted by the quartz plates is collected and transmitted to light-sensitive devices through air-core light-guides. All the light guides of the prototype were equipped with Dupond $\left[\mathrm{AlO}+\mathrm{SiO}_{2}+\mathrm{TiO}_{2}\right]$ reflective foil with the same characteristics discussed in $[4,5]$. For light collection devices we used two different types of PMTs: (i) the Hamamatsu R5380Q, and (ii) a radiation-hard multi-mesh FEU-187 from RIE St. Petersburg, with cathode area $\sim 2 \mathrm{~cm}^{2}$ [4]. The RIE phototubes were used only in the electromagnetic section of one semi-octant of the prototype, for comparison of their performance.

The electrical readout for the CASTOR calorimeter follows the design of the frontend cards used for the CMS hadronic calorimeter (HCAL), with circuits around the ASIC called QIE (charge Integrator and Encoder) [6]. This chip integrates the charge for every bunch crossing and digitizes it with a dynamic range of $10^{4}$ and with a quantization error below $3 \%$. One of the principal requirements for the readout is the ability to cover a wide dynamic range, for energy depositions from minimum ionizing particles (MIPs) to showers of particles with energies of the primary beam. This is accomplished with the QIE cards, through a multirange technique. The sensitivity to MIPs is very important for the calibration with halo muons of the proton beams. Since the occupancy is expected to be high, the electronics have to separate signals within the LHC bunch to bunch time of $25 \mathrm{~ns}$. The QIE uses four capacitors to accumulate the charge in $25 \mathrm{~ns}$ time samples. Each subsequent time sample comes from a different capacitor. For every triggered event, during the test beam, there were twenty time samples. The sum of two of them was used to reconstruct the calorimeter's signals. A typical pulse shape distribution is shown in Fig. 2.

\section{Beam test 2007}

The results of the beam test and simulation studies with CASTOR prototype I [4] and prototype II [5] allowed us to define a full-length prototype with (quasi)final characteristics. The beam tests were carried out in the $\mathrm{H} 2$ beam line at CERN Super Proton Synchrotron (SPS) during two weeks in August-September 2007. The tested prototype was one full-length octant, consisting of electromagnetic (EM) 


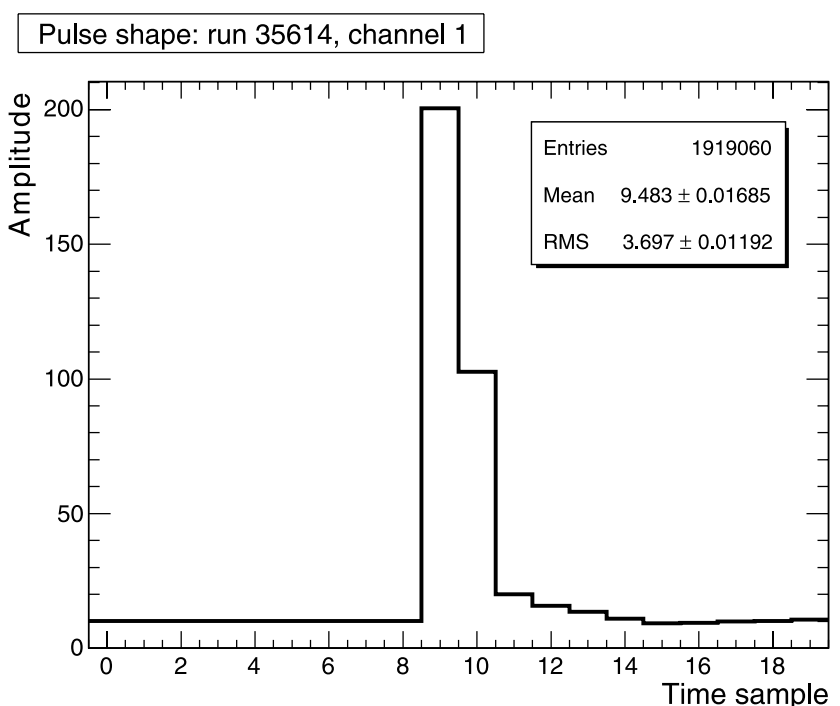

Fig. 2 Typical pulse shape obtained with an electron beam. The signal is sampled in twenty time slices, for every triggered event, and the sum of two of them was used to reconstruct the calorimeter's response

JURA

\begin{tabular}{c|}
$\qquad$\begin{tabular}{|l|l|l|l|l|l|l|l|l|l|l|l|l|l|}
\hline 2 & 4 & 2 & 4 & 6 & 8 & 10 & 12 & 14 & 16 & 18 & 20 & 22 & 24 \\
\hline 1 & 3 & 1 & 3 & 5 & 7 & 9 & 11 & 13 & 15 & 17 & 19 & 21 & 23 \\
\hline
\end{tabular} \\
SALM \\
SALVE
\end{tabular}

Fig. 3 Schematic drawing of the beam-tests-2007 CASTOR prototype with the 28 readout units (RUs) indicated. The beam comes from the left impinging on the EM sections

and hadronic (HAD) sections, with a total of 28 channels (Figs. 3, 4). A schematic view of the $\mathrm{H} 2$ beam line is shown in Fig. 5. Electron, pion and muon beams of several energies were available, and the energy response, in terms of the linearity and resolution, was obtained through energy scans. The prototype was placed on a platform movable with respect to the beam in both horizontal and vertical directions (Fig. 4). Wire chambers were installed upstream of the pro-

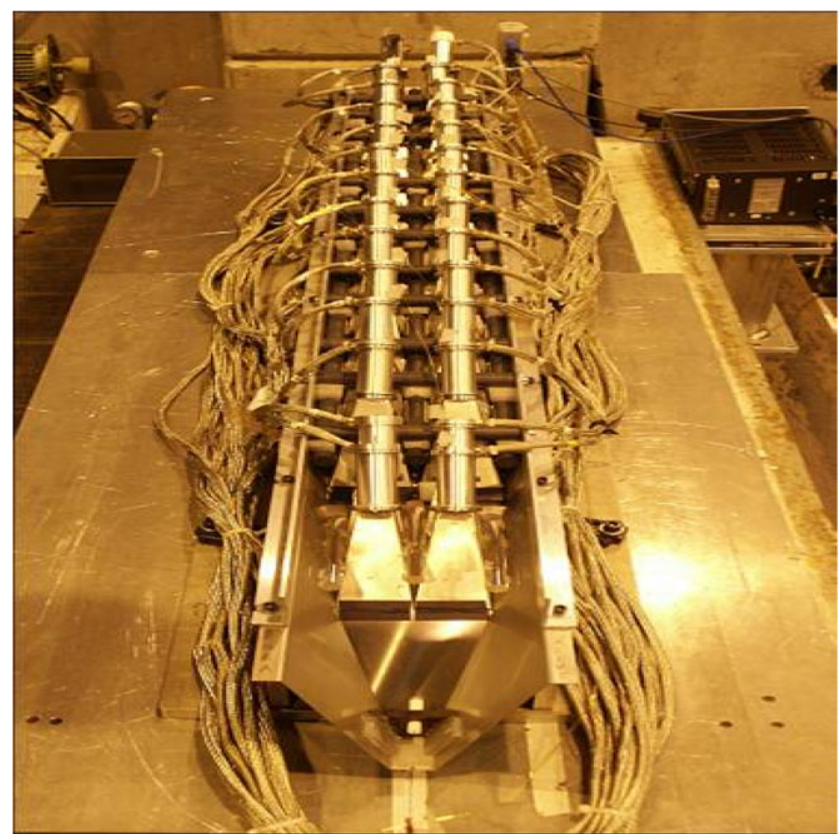

Fig. 4 Photograph of the fully instrumented CASTOR octant prototype installed on top of the moving table at the CERN/SPS H2 line (Aug. 2007)

totype (Fig. 5), giving precise information on the position of each particle hitting the calorimeter. In this way, the beam profile was known so that particular regions of the profile could be selected for the analysis of the calorimeter's spatial response. Figure 6 shows the two semi-octants of the electromagnetic (black) and of the hadronic (red) sections, as seen projected onto a plane at $45^{\circ}$ with respect to the beam axis. Due to the different sizes of the W/Q-plates there is not complete overlap of the two sections. The horizontal and vertical numbers correspond to distances, along the plate, of the points used for the horizontal and vertical position scans.

\subsection{Pedestal stability}

The stability of pedestals, calculated using two time samples for the 28 channels of the calorimeter, was studied as a func-

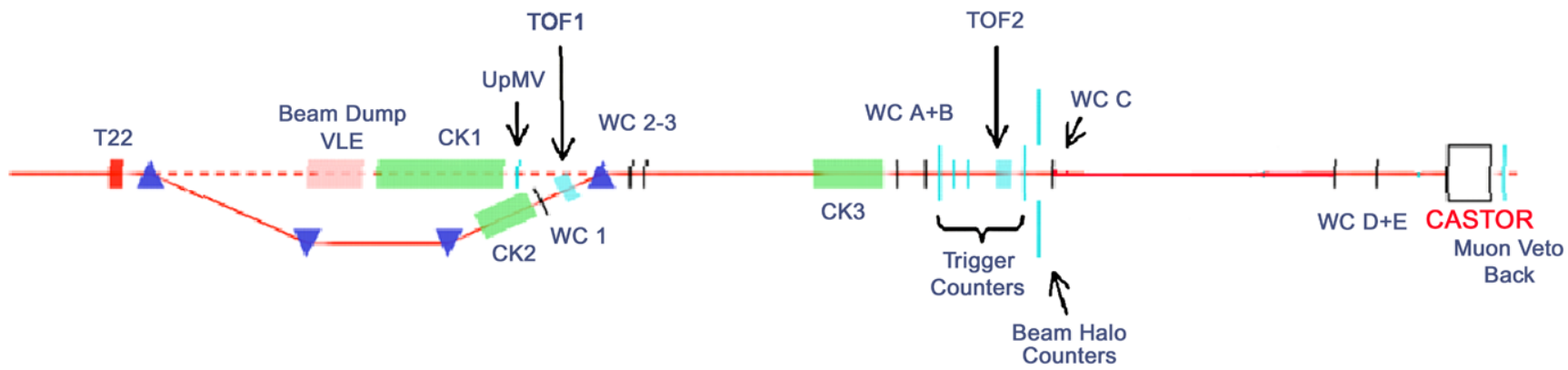

Fig. 5 Schematic figure of the $\mathrm{H} 2$ beam line of the SPS at CERN 


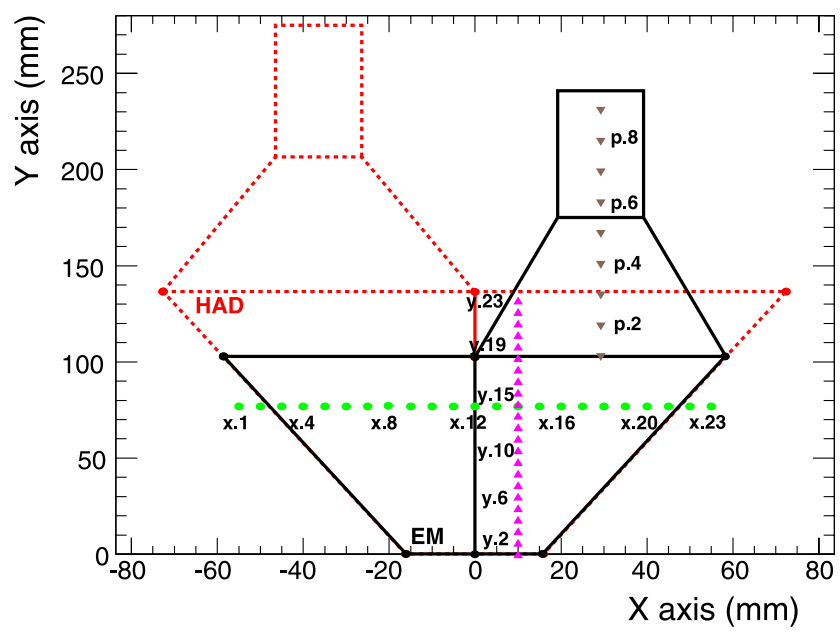

Fig. 6 Projection of the EM (solid black lines) and HAD (dashed red lines) sections onto a $45^{\circ}$ plane. The beam impact points used in the horizontal and vertical scans are shown

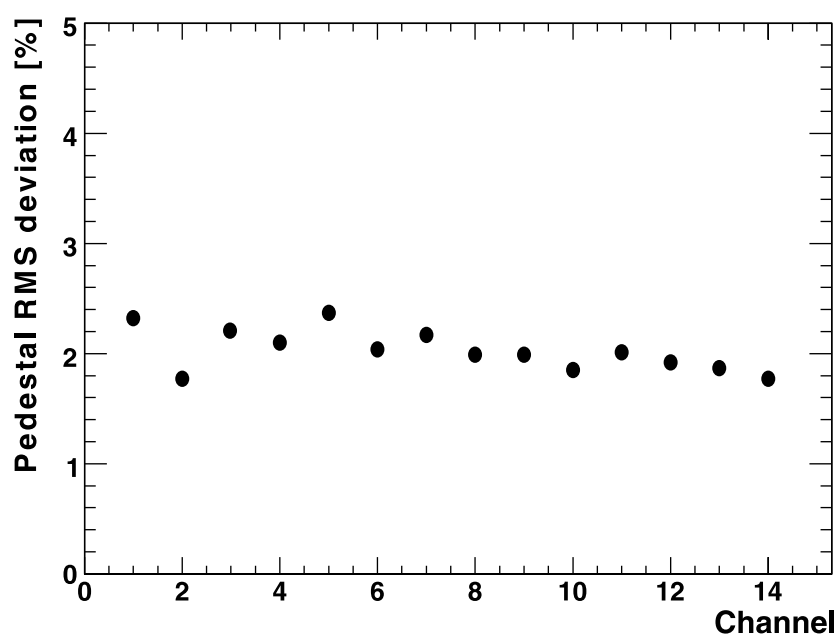

Fig. 7 Deviation of the pedestal RMS value during the entire period of the test beam, for all 14 channels of the semi-octant equipped with the Hamamatsu photomultipliers

tion of time. Figure 7 shows the pedestal RMS deviation for all channels of the semi-octant that was equipped with the Hamamatsu photomultipliers. The selected runs cover the whole time period of the test beam and refer to electron, pion and muon beams of various energies, considering pedestal trigger events. The noise is found to be stable within approximately $2 \%$. Figure 8 shows the variation of the pedestal mean value for the first channel of the EM section of the calorimeter, as well as the variation of the pedestals per channel for each of the four capacitors of the QIE card. The data are well fitted with a constant value: for all channels the variation of the pedestal amplitudes is within $1 \%$ of their average value. Similar stability was observed for the channels equipped with the RIE FEU187 PMTs. Overall, the results demonstrate the stability of the pedestals mean value and RMS, during the entire time period of the test beam.

\subsection{Event selection criteria}

Information from various beam-line counters was used in order to further investigate the quality of the data and to select events without contamination from unwanted particles. The ADC counts from four trigger counters (S1, S2, S3, S4) were exploited to tag single particle events. The wire chambers hits were examined for the selection of a focused beam, while muons were tagged using the ADC information of a muon veto counter (MVB) placed behind the CASTOR prototype. For the rejection of hadrons in electron beams, the signals in the hadronic channels of the calorimeter were exploited.

The trigger counters (S1 through S4) installed along the $\mathrm{H} 2$ beam line are polystyrene scintillators of $1 \mathrm{~cm}$ thickness, hence all beam particles behave as minimum ionizing. A coincidence between a subset of the counters was used as trigger. Signal particle events were selected fitting the first peak of the pulse height distribution in ADC counts (see Fig. 9) with a Gaussian function. Events beyond $3 \sigma$ from the mean value were rejected. Delay-line wire chambers (WCA through WCE) were available to define the position of the beam particles on the front face of the calorimeter. The chamber's hit positions were requested to be within a small circular region of the usually wide beam spot, with a typical radius of $\sim 3 \mathrm{~mm}$ (see Fig. 14).

Energetic muons were tagged with a veto scintillator counter (MVB), placed behind the calorimeter. An $80 \mathrm{~cm}$ thick iron block was inserted in front of the counter. Figure 10 shows a typical pulse height distribution of the muon counter, as well as the Gaussian fit which was used to obtain the mean and width of the spectrum. Events within $3 \sigma$ with respect to the peak position of the ADC signal distribution were treated as muons and were rejected.

\subsection{Response to muons and intercalibration}

The response of the calorimeter to muons was studied with 50 and $150 \mathrm{GeV}$ muon beams. Figures 11, 12 show the muons' signal distributions measured for the $150 \mathrm{GeV}$ beam with two different PMTs: Hamamatsu R5380Q, and RIE FEU187. In both cases, the muon peaks can be fitted by a convolution of Landau and Gaussian distributions and are separated from the pedestal. From the peak position and pedestal's RMS value, the signal to noise ratio was found to be $\mathrm{S} / \mathrm{N} \approx 7$ and $\mathrm{S} / \mathrm{N} \approx 3$ for the Hamamatsu and RIE PMTs respectively. We concluded that the Hamamatsu R5380Q PMT performs better in identifying the muon signal above the pedestal. 
Fig. 8 Left: Typical plot of the pedestal mean amplitude in ADC counts as a function of the run number, corresponding to the first EM channel (EM1). The corresponding time period of the test beam is displayed in the upper horizontal axis. Right: Typical plot of the variation of the pedestal amplitudes per channel for each of the four capacitors of the QIE cards

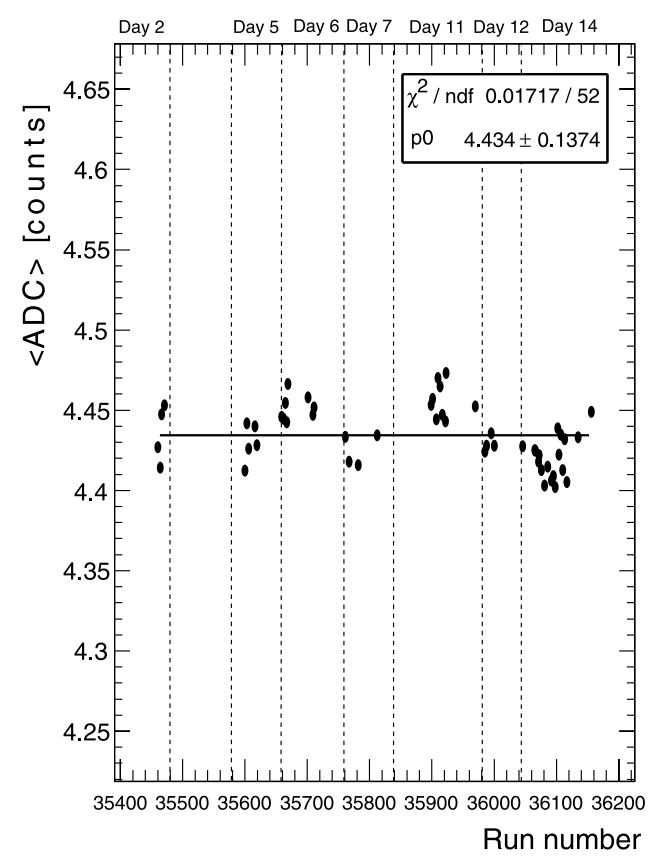

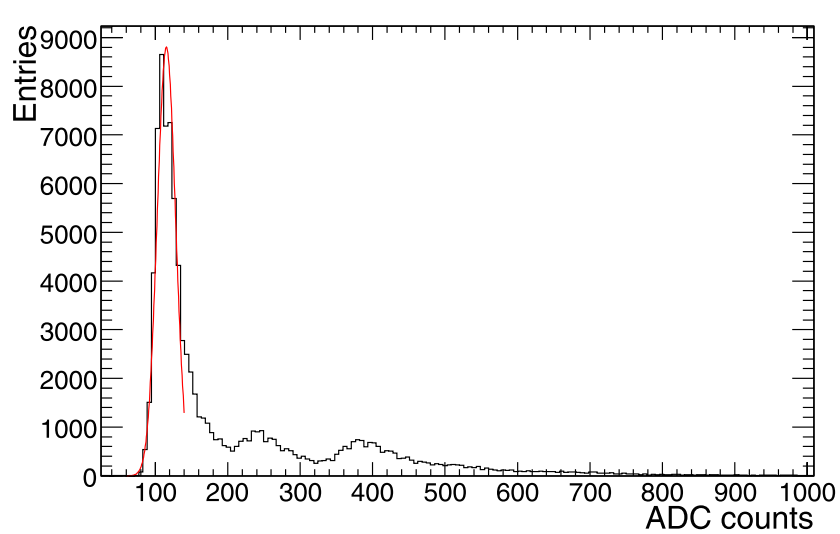

Fig. 9 Typical ADC distribution of a scintillator trigger counter (S1). The data are fitted by a Gaussian and events within $3 \sigma$ are selected as single particle events

For the initial test beam setup, in the hadronic channels of the prototype, there was no clear separation of the muon signal from the pedestal. The reason was the fact that in the initial design the light-exit surface of the quartz plates was cut at $45^{\circ}$ with respect to the axis of the quartz plate. For the EM channels, this angle was $90^{\circ}$. Due to total reflection of the Čerenkov light at the exit surface of the hadronic channels, the light collection efficiency was considerably reduced. At a later stage of the test beam, one of the hadronic channels was equipped with a set of $90^{\circ}$-cut quartz plates, leading to an improvement of the efficiency by a factor of $\sim 1.5$.

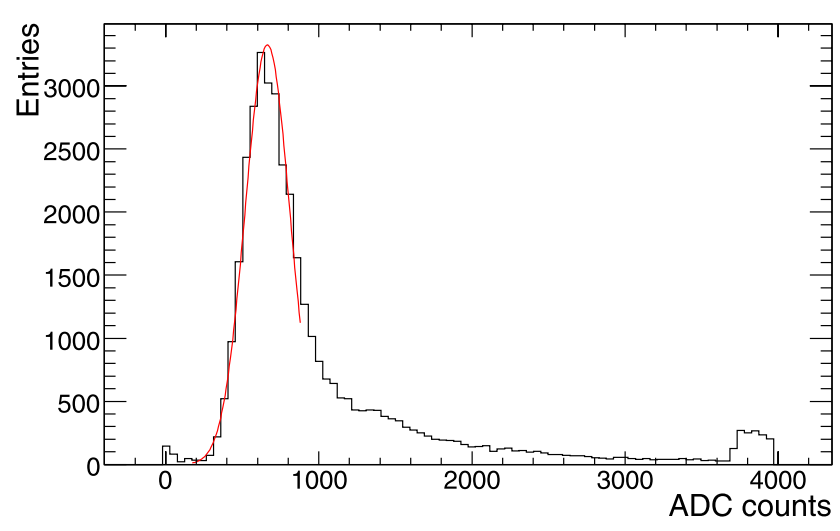

Fig. 10 Typical example of the ADC distribution of the muon veto counter, for a run with a muon beam with $E=150 \mathrm{GeV}$

The good pedestal-mip separation allowed us to use the muon signal distributions for channel intercalibration. The intercalibration constant per channel was derived from the following formula:

$F_{i}=E_{1} / E_{i} \times C_{\text {absorber }} \times C_{\mathrm{QP} \text { cut }}$,

where $E_{i}$ is the muon signal of the calorimeter in ADC counts for channel $i$,

$C_{\text {absorber }}= \begin{cases}1, & \text { for } \mathrm{EM}, \\ 2, & \text { for HAD }\end{cases}$ 
Fig. 11 Signal distribution measured in one of the channels of the EM (left) and HAD (right) sections of the calorimeter, for muons of $150 \mathrm{GeV}$ energy impinging on the right semi-octant equipped with Hamamatsu PMTs. The pedestal distribution is also shown for comparison. The muon signal was fitted with a convolution of Landau and Gaussian distributions, obtaining the fit parameters reported in the upper inset
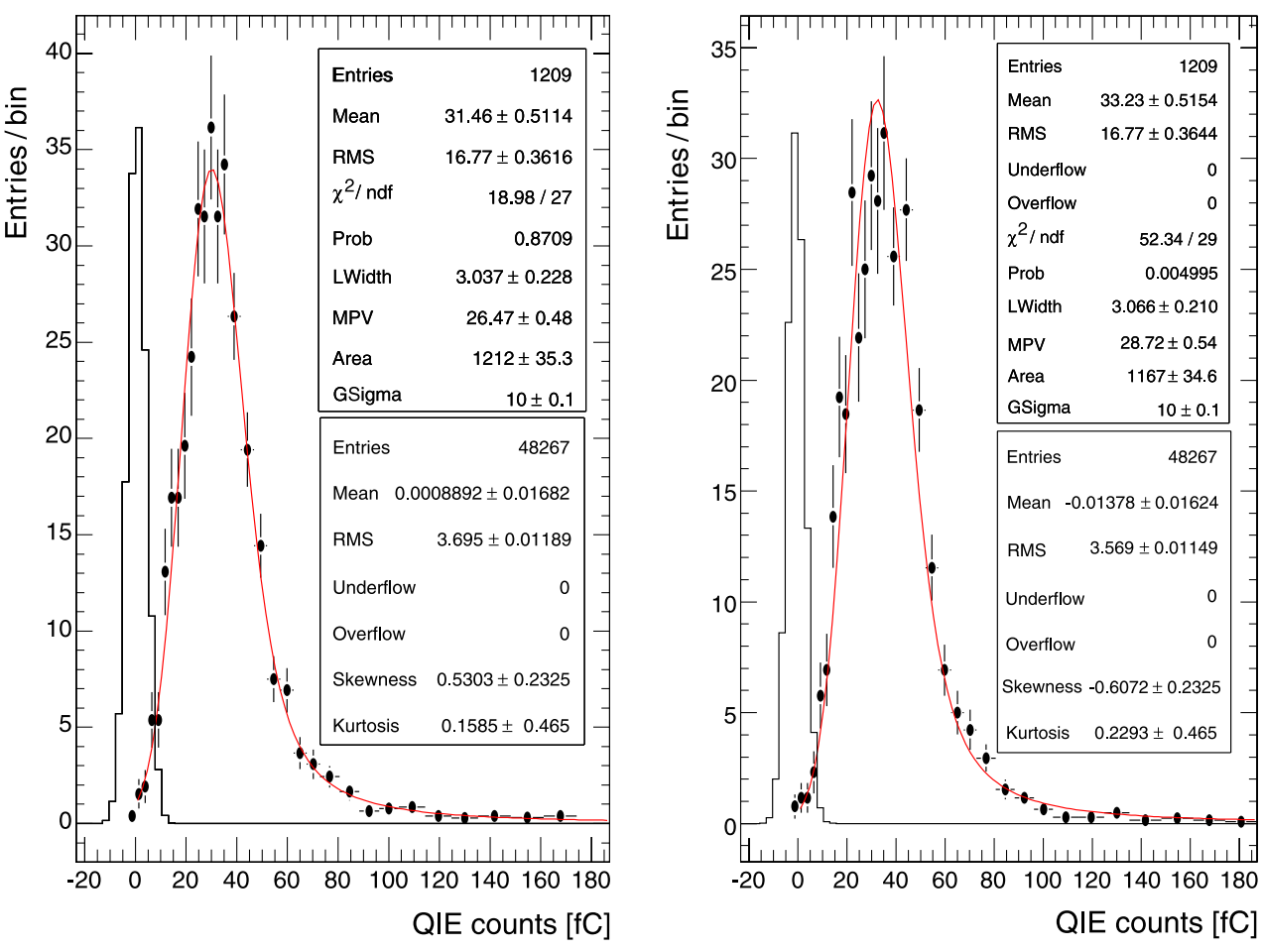

Fig. 12 Signal distribution measured in the channels of the EM section of the calorimeter for muons of $150 \mathrm{GeV}$ energy impinging on the left semi-octant equipped with RIE FEU187 PMTs. The pesestal distribution is also shown for comparison. The muon signal was fitted with a convolution of Landau and Gaussian distributions, obtaining the fit parameters reported in the upper inset

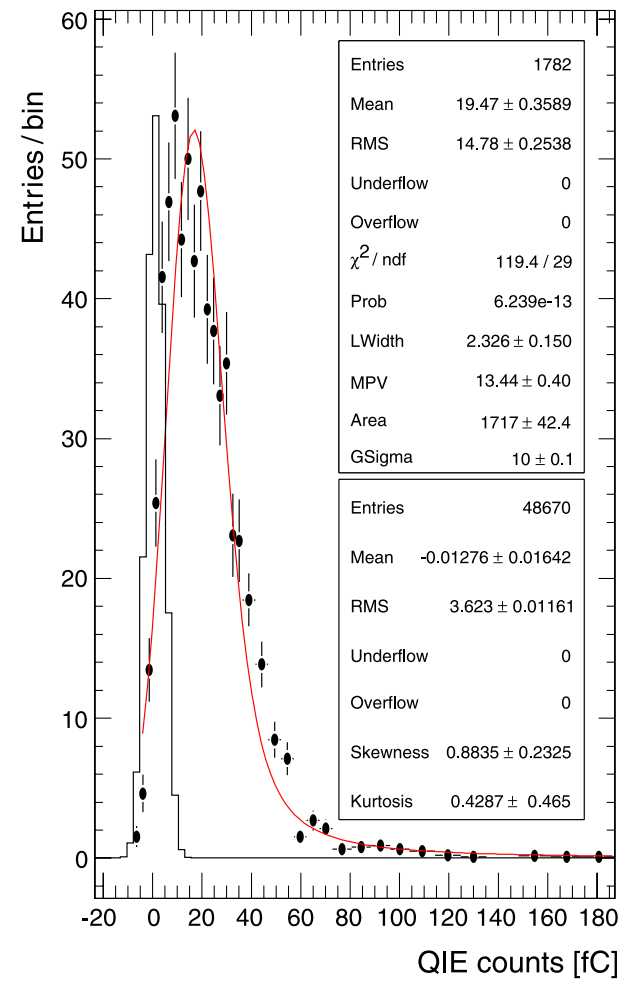

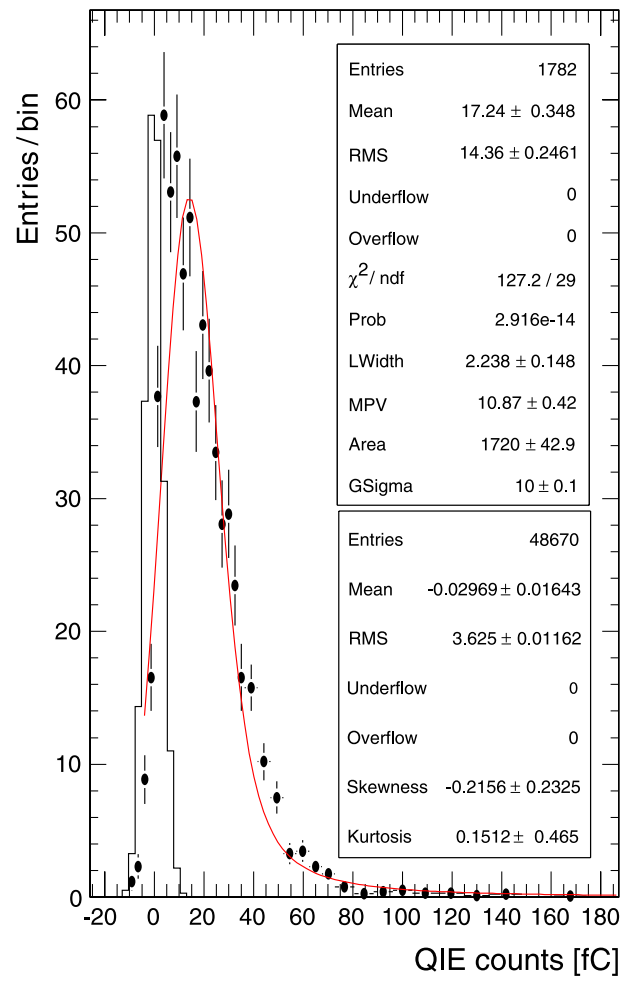

and

$C_{\mathrm{QP} \text { cut }}=\left\{\begin{array}{l}1, \\ \quad \text { for channels with Q-plates cut at } 90^{\circ}, \\ G(\pi) / G(\mu), \\ \quad \text { for channels with Q-plates cut at } 45^{\circ},\end{array}\right.$ where $C_{\text {absorber }}$ accounts for the double thickness of the hadronic section's plates and $G(\pi), G(\mu)$ is the light gain for the transition after the change of the Q-plates for pions and muons respectively. Statistical uncertainties were calculated through error propagation in (1), assuming independent measurements in each channel, i.e. uncorrelated mea- 
Fig. 13 Energy distribution for muons of 50 (left) and $150 \mathrm{GeV}$ (right) impinging on one semi-octant of the calorimeter

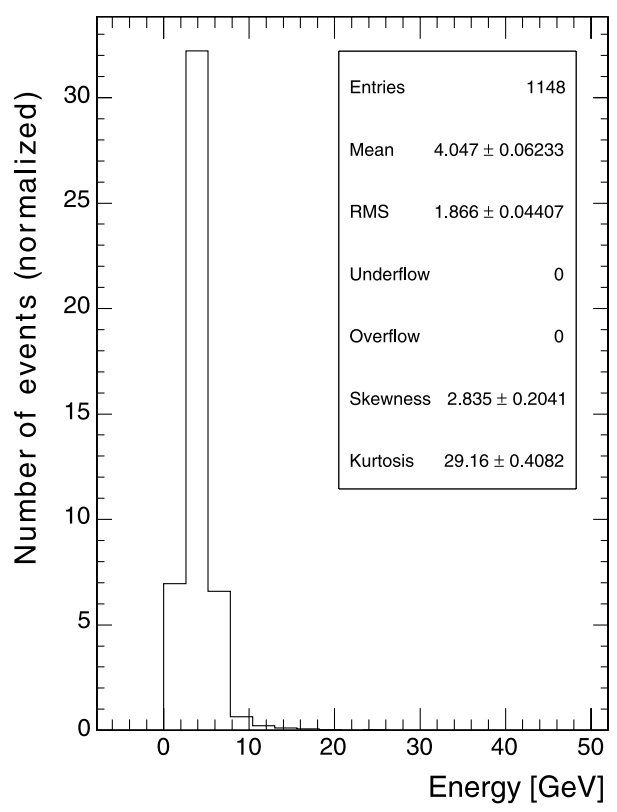

surement errors. The conversion of the collected charge to units of energy was performed on the basis of the calorimeter's calibration with electrons. Based on that conversion, and after summing the signals in one sector of the calorimeter, the mean total energy deposited was found to range from 4 to $5 \mathrm{GeV}$ for 50 and $150 \mathrm{GeV}$ muons respectively (see Fig. 13). Such high energy muons are expected to deviate from real MIPs, as they produce mini electromagnetic showers through their passage in the detector.

\subsection{Response to electrons}

Electron beams of energy from 30 to $200 \mathrm{GeV}$ were used to test the electromagnetic response linearity and resolution. Spatial properties of the electromagnetic shower were studied as well. For all energy scan studies, a central point in the calorimeter was selected while, offline, a $3 \mathrm{~mm}$ radial cut was applied on the spatial profile of the beam, as measured by the upstream wire chambers located closer to the calorimeter (see Fig. 14). The energy distributions of 50 and $200 \mathrm{GeV}$ electrons are shown in Figs. 15. To study the response of the calorimeter as a function of the energy, a central point in the semi-octants was exposed to beams of various energies. The signal distributions were very well fitted by a Gaussian function. The signal of the calorimeter, per $\mathrm{GeV}$ of deposited energy for electrons, is plotted as a function of the beam energy in Fig. 16. The calorimeter's response was found to be linear, within $4 \%$, in the energy range explored and corresponding to approximately $41 \mathrm{ADC}$ counts/GeV. The conversion of ADC counts to $\mathrm{GeV}$ was thus applied accordingly, leading to the energy distributions shown in Fig. 15.
The relative energy resolution of the calorimeter has been studied by plotting the normalized width of the Gaussian signal distributions, with respect to the incident beam electron energy, and fitting the data points with the functional form [4]:

$\sigma / E=p_{0} \oplus p_{1} / \sqrt{E}$

where the $\oplus$ indicates that the terms are added in quadrature. Two general terms contribute to the energy resolution of the calorimeter. The constant term, $p_{0}$, is related to imperfections of the calorimeter, signal generation and collection non-uniformity, calibration errors and fluctuations in the energy leakage, which limit the resolution at high ener-

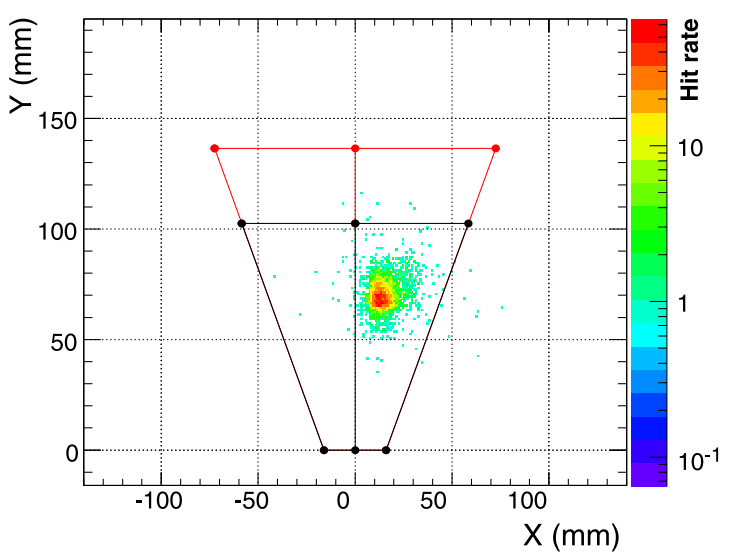

Fig. 14 Typical beam profile of $200 \mathrm{GeV}$ electrons impinging on the right semi-octant of the calorimeter, as measured by the scintillator-wire-chamber telescope upstream 
Fig. 15 Energy distribution for electrons of $50 \mathrm{GeV}$ (left) and $200 \mathrm{GeV}$ (right) entering the right semi-octant of the calorimeter
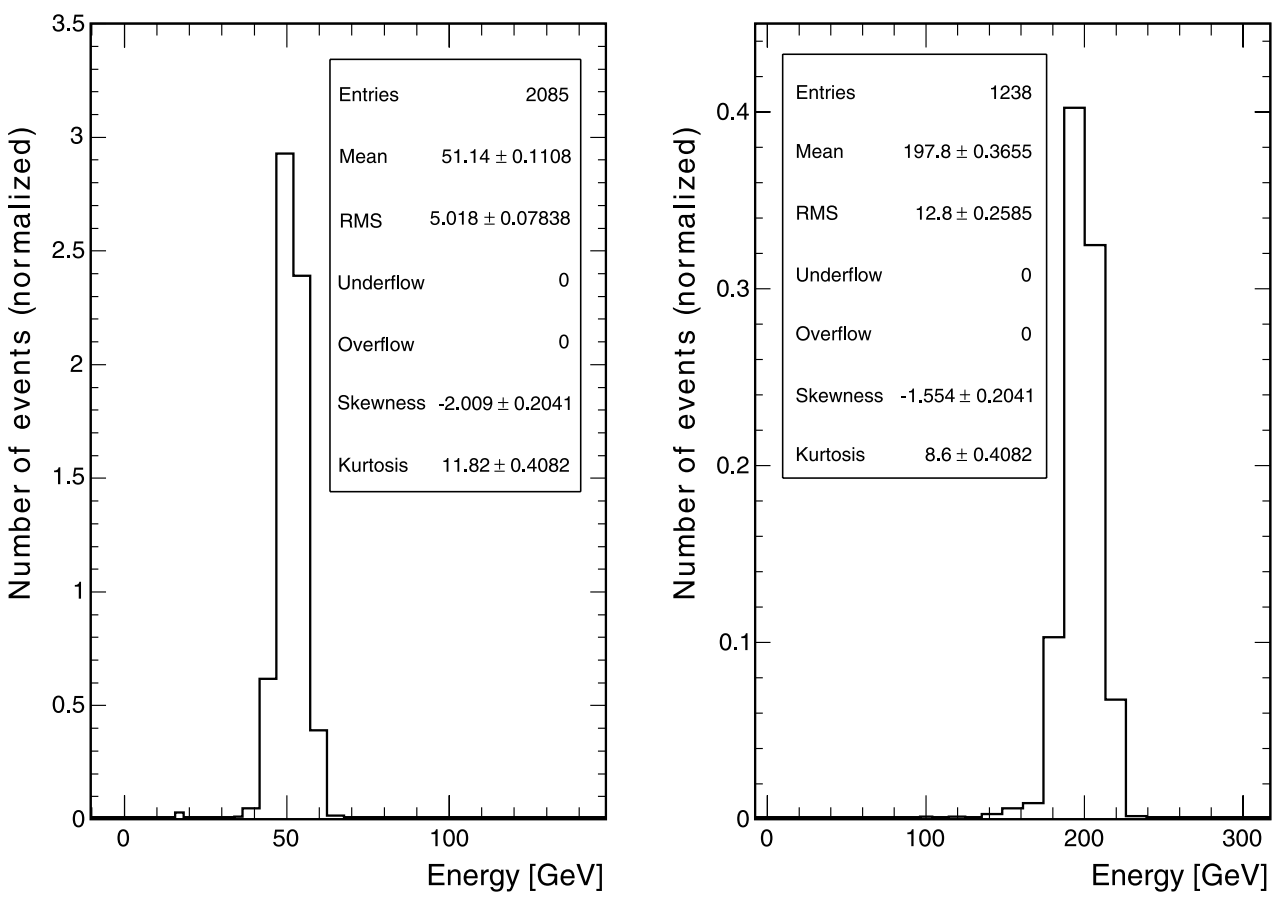

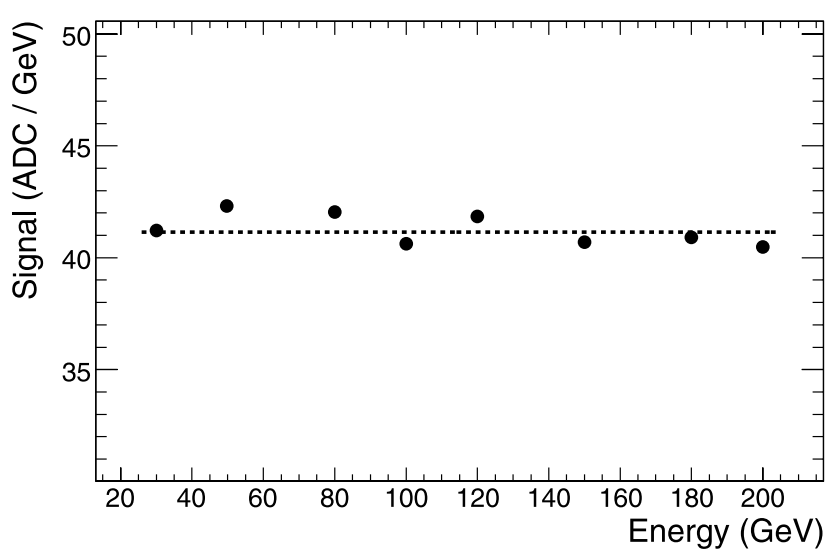

Fig. 16 Response of the calorimeter to electrons. The data points were fitted with a zero degree polynomial. The statistical uncertainties are too small to be visible

gies. The stochastic or sampling term, $p_{1}$, is due to intrinsic shower photon statistics and characterizes the fluctuations in the signal generating process. Figure 17 shows the fit to the energy resolution with expression (4). The measured stochastic term for the Hamamatsu photomultipliers was found to be $\sim 43 \%$, whereas the constant term $p_{0}$ is about $4.5 \%$.

The energy deposition of electrons in the hadronic section of the calorimeter was found to be negligible. Figure 18 shows the ratio of the mean signal in the two sections of the prototype, indicating that for energies up to $200 \mathrm{GeV}$, the fraction of the energy which escapes the electromagnetic

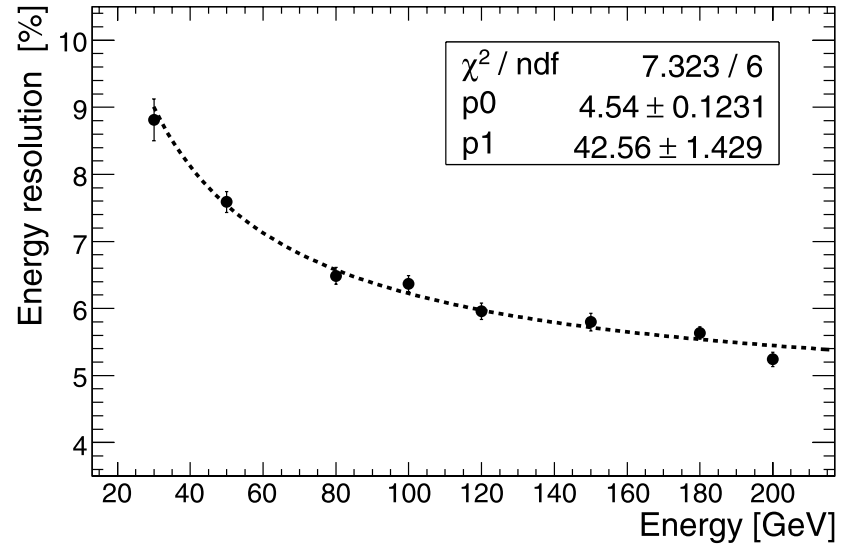

Fig. 17 Energy resolution of the prototype calorimeter, as measured with the Hamamatsu photomultipliers for electrons. The fit parameters shown in the inset are obtained with (4) while the widths of the electron signal distributions were fitted by a Gaussian function

calorimeter, and is collected in the hadronic, stays below $1 \%$.

\subsection{Response to pions}

Pion beams of energy from 20 to $300 \mathrm{GeV}$ were available for the study of the response of the calorimeter to hadrons. The total CASTOR depth is $10 \lambda_{I}$, taking into account that the two first electromagnetic channels effectively act, in depth, as part of the hadronic section. Typical energy distributions, obtained with high energy pions incident on the prototype, 
are shown in Fig. 19, where the signals in both EM and HAD sections of the calorimeter were summed. The hadronic energy linearity and resolution were thus obtained. Figure 20 shows the linearity of the CASTOR prototype to incident pions as obtained by measuring the total energy deposited in the calorimeter sections and correlating the mean value of the signal distributions with each corresponding beam energy. The non linearity of the response at lower energies, reflects the non-compensating character of the detector. At energies above $100 \mathrm{GeV}$, the mean signal is constant within $5 \%$ and corresponds to approximately 20 ADC counts/GeV.

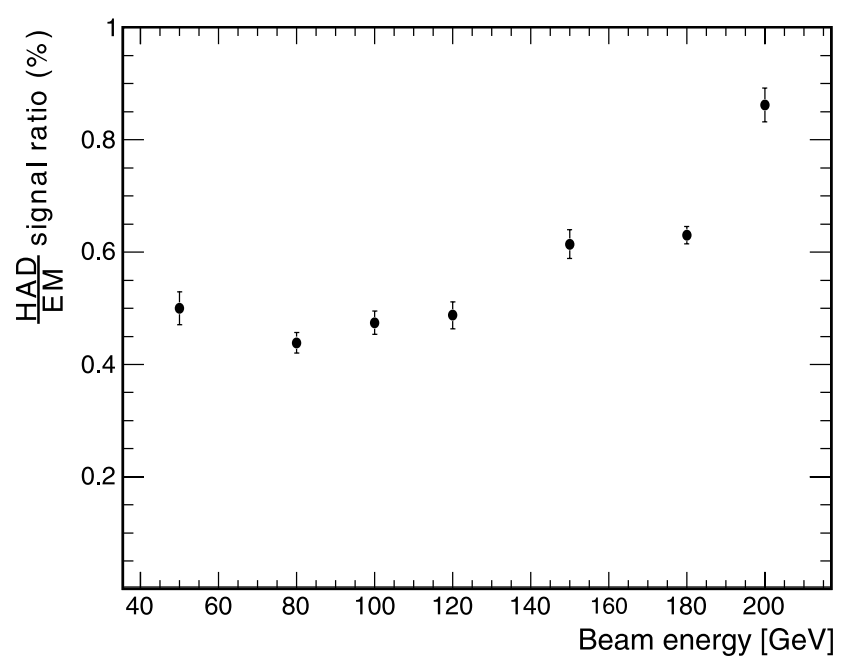

Fig. 18 The ratio of the mean signal in the hadronic and electromagnetic sections of the calorimeter for incoming electrons
The conversion of ADC counts to GeV was thus applied, leading to the energy distributions shown in Fig. 19.

The relative energy resolution of the calorimeter, shown in Fig. 21 and fitted with the functional form (4), was derived from the mean and RMS values of the signal distributions. A constant term, p0, of 18.3 and a stochastic term, $\mathrm{p} 1$, of 187 were obtained. The resolution is found to be significantly better with respect to the 2004 beam test [5]. The improvement of the resolution is expected, as the prototype tested in 2007 consists of a full hadronic sector, i.e. a total of $\sim 10$ interaction lengths, while the second one was only 4.26 interaction lengths, although no detailed comparison can be made due to the differences in the specifications of the two prototypes. The collected energy in the EM section with respect to the total hadronic energy, for a pion beam, is shown in Fig. 22.

The energy deposition of pions in the electromagnetic section is significant. Figure 22 shows the ratio of the mean signal in the two sections of the prototype, indicating that for energies below $80 \mathrm{GeV}$, the fraction of the energy which is collected in the electromagnetic calorimeter is above $10 \%$.

\subsection{Uniformity of the response}

The uniformity of the calorimeter's response was studied with position scans. Figure 23 presents results of a twodimensional scan with a muon beam of $150 \mathrm{GeV}$. The mean values of the signal are shown for the first four longitudinally ordered channels of both semi-octants of the prototype. The signal is maximal at the lowest and minimal at the
Fig. 19 Energy distribution for pions of $100 \mathrm{GeV}$ (left) and 180 $\mathrm{GeV}$ (right) entering the right semi-octant of the calorimeter
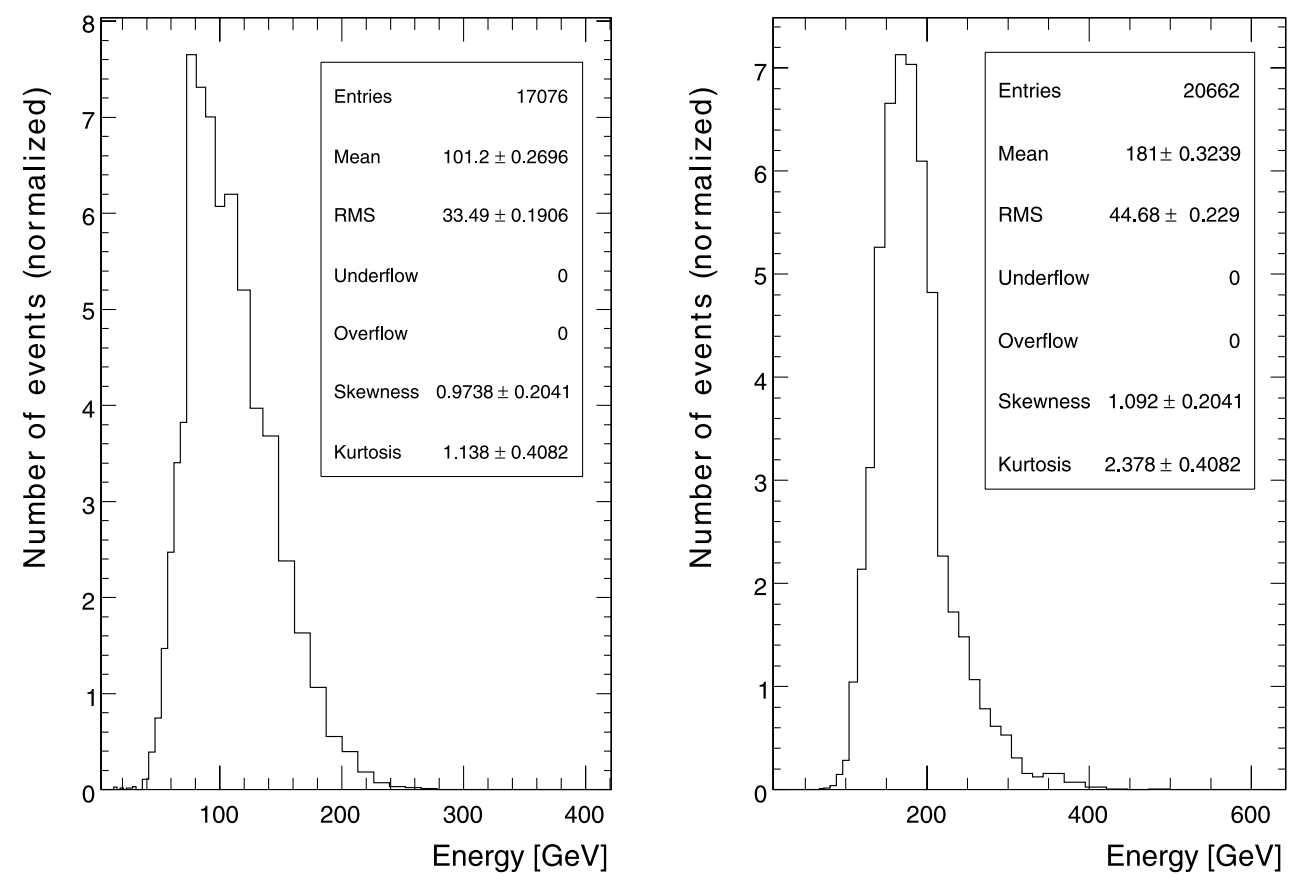


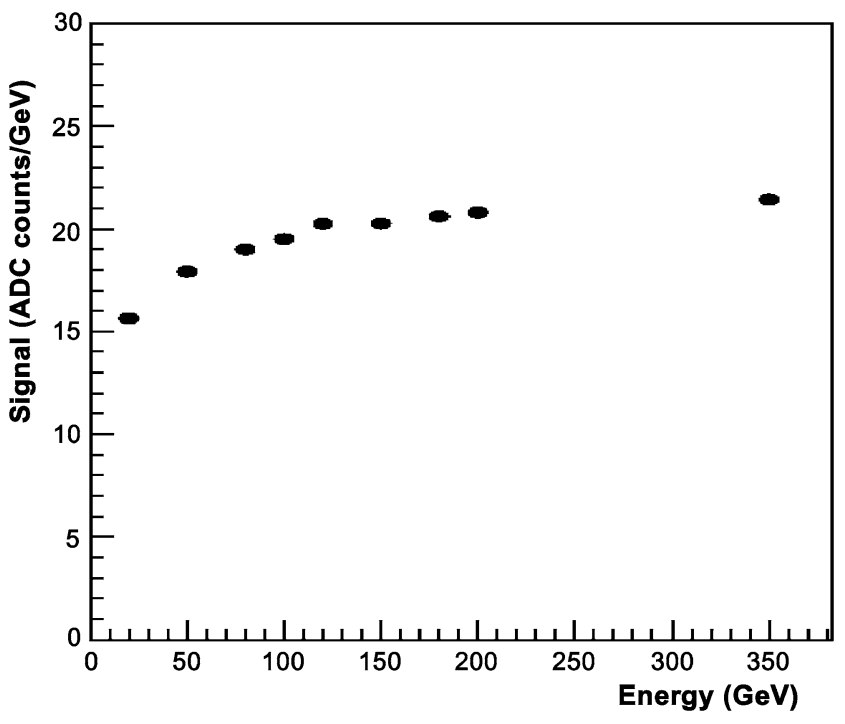

Fig. 20 Response of the calorimeter to pions of various energies, obtained with Hamamatsu PMTs. The statistical uncertainties are too small to be visible

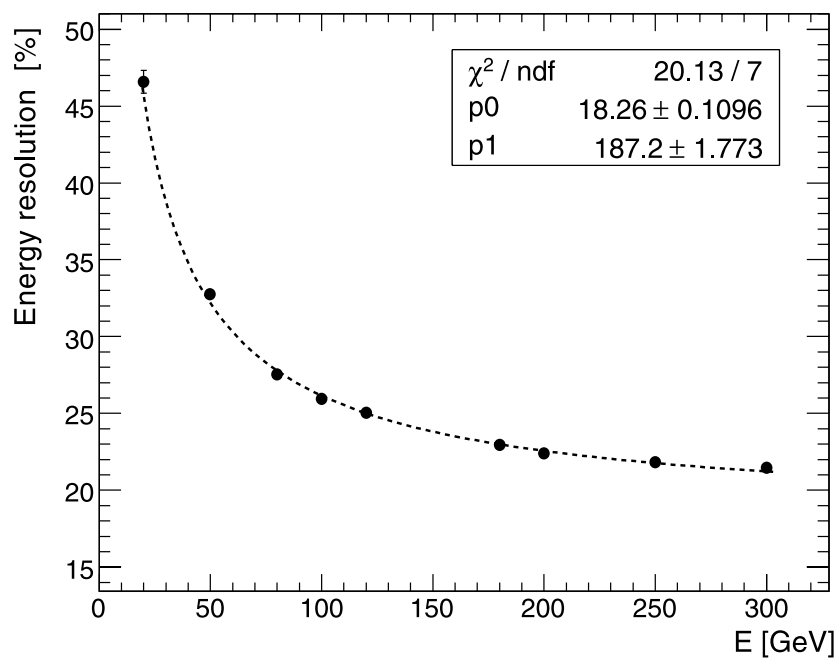

Fig. 21 Energy resolution of the CASTOR prototype calorimeter to pion beams of several energies obtained with the Hamamatsu PMTs. The fit parameters shown in the inset are obtained with (4)

highest $y$ position, as well as at the lowest absolute values of $x$.

Figure 24 (left) presents the results of the horizontal scan at a fixed vertical position (within a $5 \mathrm{~mm}$ wide band around $y=75 \mathrm{~mm}$ ) for the muon beam. The mean signal in the first six successive sections in two semi-octants of the prototype is shown. The signal distribution reveals nonuniformity in the electromagnetic section, while it is uniform in the hadronic channels. The behavior is more complicated for other vertical positions, as can be seen in Fig. 24 (right) where the results of the horizontal scan at different $y$-positions are shown for one sector. For the electromagnetic sections the non-uniformity appears to be maximal at

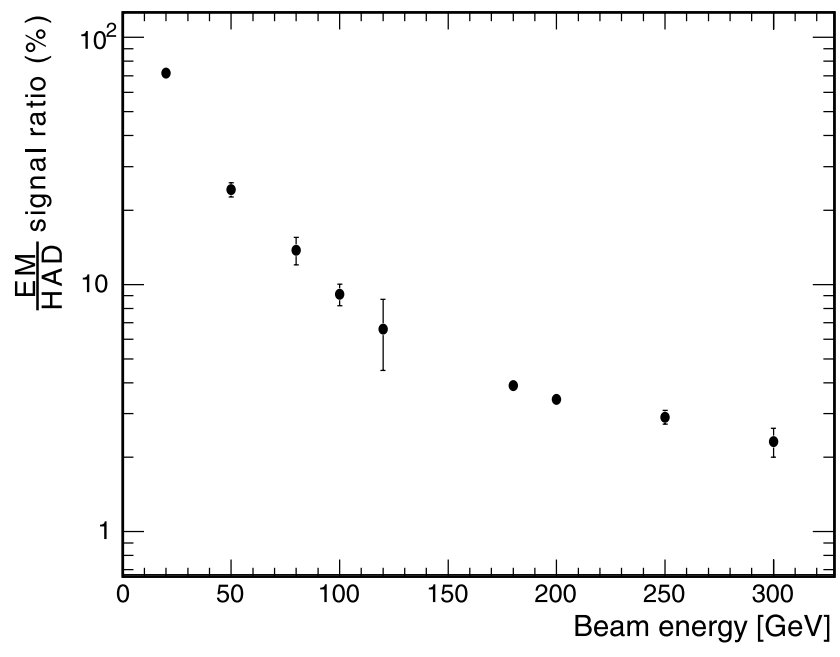

Fig. 22 The ratio of the mean signal in the electromagnetic and hadronic sections of the calorimeter for incoming pions

$y=70 \mathrm{~mm}$ while for the hadronic sections non-uniformity appears for channels 4-6 at the highest $y$-position. Figure 25 presents results of the vertical scan. The mean signal in channels $1-6$ is shown for different horizontal positions. The response in the electromagnetic section demonstrates nonuniformity, while in the hadronic section, non-uniformity is observed at $y>70 \mathrm{~mm}$. The signal in the first hadronic channel, equipped with the quartz plates' cut at a $90^{\circ}$ angle at the exit surface, was found to be more uniform.

The uniformity of the response was also studied with electron and pion beams. Figure 26 shows the total signal in the electromagnetic and hadronic sections for a horizontal scan at several vertical positions on the front face of the calorimeter, with electrons and pions of $80 \mathrm{GeV}$. The largest non-uniformity of the response to electrons was found for $y \approx 75 \mathrm{~mm}$, while at high values of $y$ the mean signal is uniform. The response to pions is in general more uniform. Non-uniform behavior is seen for pions only at relatively high values of $y$.

\subsection{Shower properties}

\subsubsection{Transverse profile}

Position scans made it possible to obtain information on the transverse profile of the electron and pion showers. Figure 27 presents a scan over $x$ at $y=75 \pm 5 \mathrm{~mm}$ for $80 \mathrm{GeV}$ electrons and pions. The longitudinally integrated signals in the left and right semi-octant, as well as their sum are shown. A significant non uniform variation over $x$ is evident for electrons which implies non-uniformity of the electromagnetic section of the calorimeter, since it is the one which contains almost the total energy of the electrons' shower. The same measurement for pions demonstrates better uniformity 

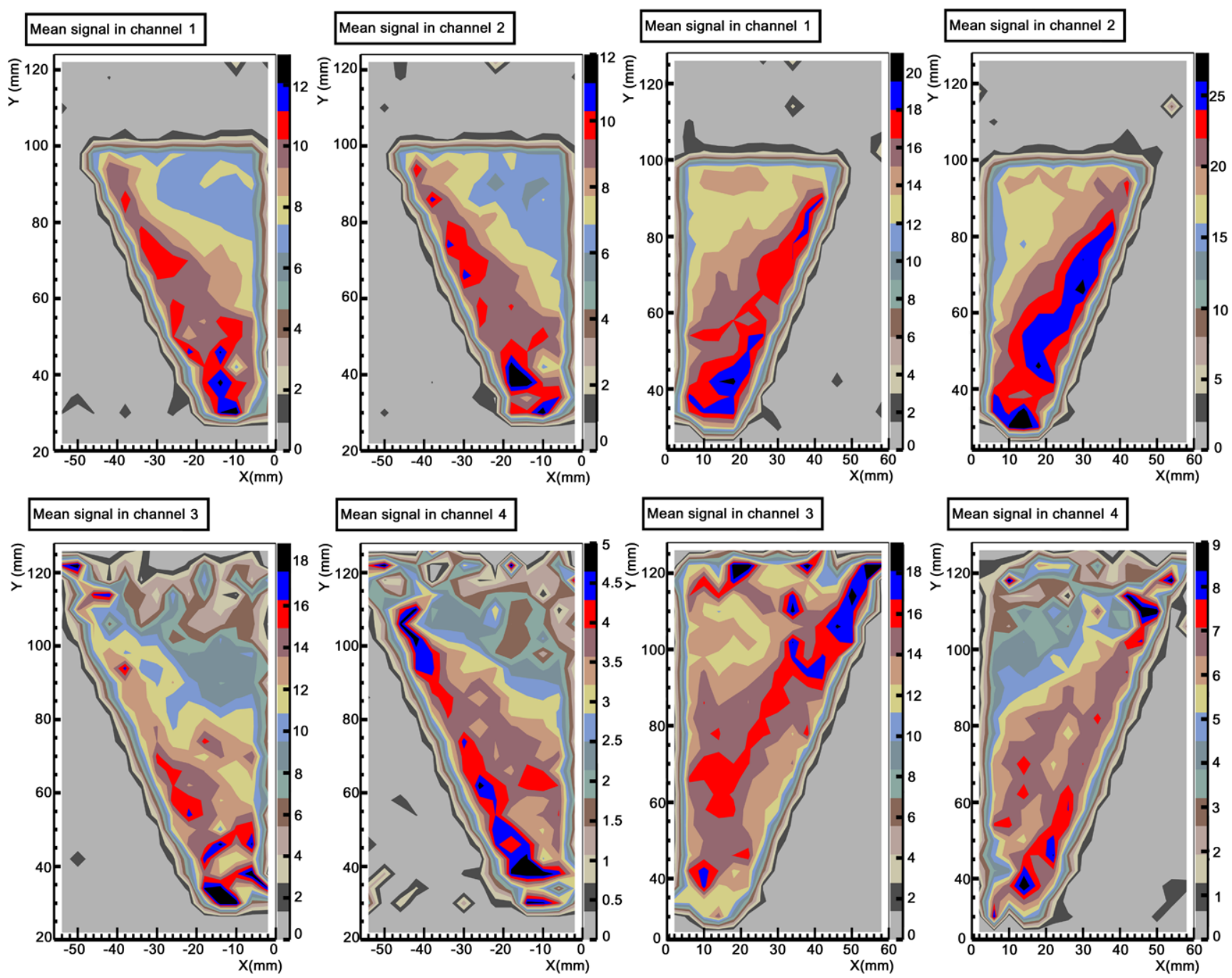

Fig. 23 Horizontal and vertical scan of the prototype with muons. The mean signal in the first four channels is shown for both semi-octants

over the horizontal position. This is a consequence of much better uniformity over $x$ in the hadronic section, where most of the energy of the hadronic showers is deposited.

From these distributions the differential distributions over $x$ were extracted, shown in Fig. 28 for electrons and pions. The distributions were fitted with use of two parameterisations, both consisting of two terms describing the core and the tail of the shower respectively. The first one is the derivative of the sigmoid parameterisation applied for the integral distribution:

$\frac{1}{E} \int_{-\infty}^{x} \frac{d E}{d x} d x=p \frac{1}{1+e^{-A_{C} x}}+(1-p) \frac{1}{1+e^{-A_{T} x}}$.

The basis of the second one is the Grindhammer parameterisation of the radial distribution [7]:

$$
\frac{1}{E} \frac{d E}{d r}=p \frac{2 r R_{C}^{2}}{\left(r^{2}+R_{C}^{2}\right)^{2}}+(1-p) \frac{2 r R_{T}^{2}}{\left(r^{2}+R_{T}^{2}\right)^{2}}
$$

which was transformed to the planar geometry of the measurements for the prototype. The two fits differ only at the very tails of the distribution, with the difference being practically unresolvable in the presented plots. The full width at half maximum equals to $\mathrm{FWHM}=4 \mathrm{~mm}$ for electrons and FWHM $=5.8 \mathrm{~mm}$ for pions. The planar shower containment, i.e. the fraction of the energy measured at $x$ ranging from $-\infty$ to a given value is shown in Fig. 29 for electrons and pions. Analytical transformation between axial and planar geometry is possible only for the Grindhammer parameterisation. Figure 30 presents the radial containment within this parameterisation for the electromagnetic and hadronic showers.

\subsubsection{Longitudinal profile}

The longitudinal segmentation and large depth of the calorimeter can provide valuable information on the longitudinal 

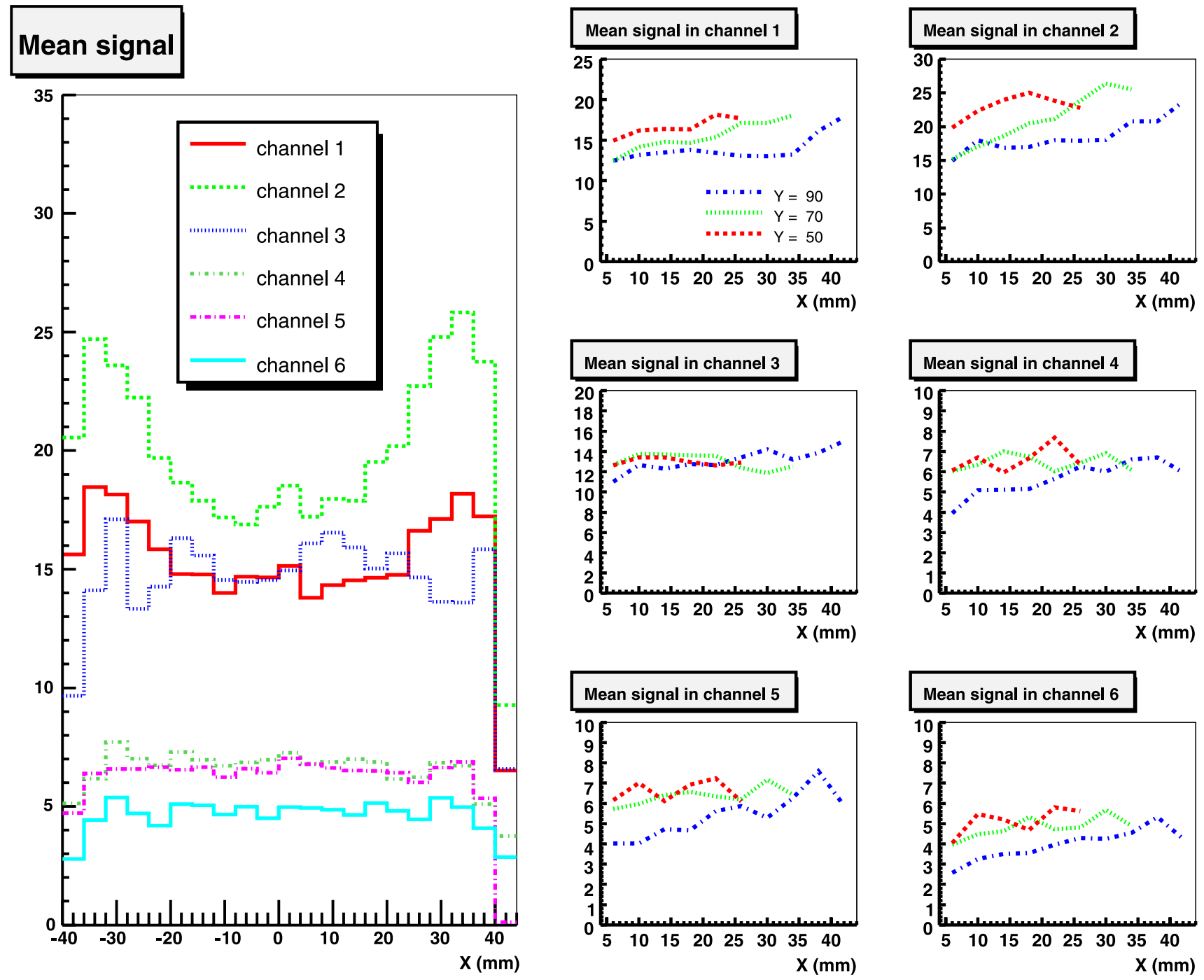

Fig. 24 Left: The mean signal of the calorimeter in channels 1-6 (longitudinally ordered), as obtained for a horizontal scan with muons, at a fixed vertical position. Right: The mean signal in channels $1-6$ for a horizontal scan with muons at different vertical positions

profile of the hadronic showers. Figure 31 presents the longitudinal profile for pions of different energies, ranging from 20 to $350 \mathrm{GeV}$. Plotted is the mean signal at different depth (measured in interaction length), normalized to the thickness of the corresponding absorber layer, and divided by the energy of the pion beam. The results indicate a fairly good scaling over energy, except for the very beginning of the cascade. The clear exponential shape of the tail is also evident.

\section{Summary}

We have presented results on the performance study of the response of a prototype of the CASTOR quartz-tungsten calorimeter for the CMS experiment. The results have been obtained from beam tests at the CERN-SPS in 2007 with high energy electrons, pions and muons. The main conclusions of this study can be summarized as follows:

1. The Hamamatsu R5380Q photomultiplier performed better than the RIE FEU187 in terms of separation of the muon signals from the pedestal signals. This feature was essential for the intercalibration of the calorimeter's channels.

2. The response of the calorimeter to electrons was found to be linear in the energy range of the tests, while the energy resolution to electromagnetic showers is characterised by a stochastic term of $\sim 43 \%$. The constant term is around $4.5 \%$.

3. The non compensating character of the calorimeter is reflected in the non linearity of the response to pions at 
Fig. 25 Mean signal in channels 1-6 (longitudinally ordered) of the prototype for a vertical scan with muons at different $x$-positions
Fig. 26 Left: Uniformity of the response to $80 \mathrm{GeV}$ electrons.

The mean total signal in the electromagnetic section is shown as a function of $x$ for different values of the vertical position $y$. Right: Uniformity of the response to $80 \mathrm{GeV}$ pions. The mean total signal in the hadronic section is shown as a function of $x$ for different values of the vertical position $y$
Mean signal in channel 1

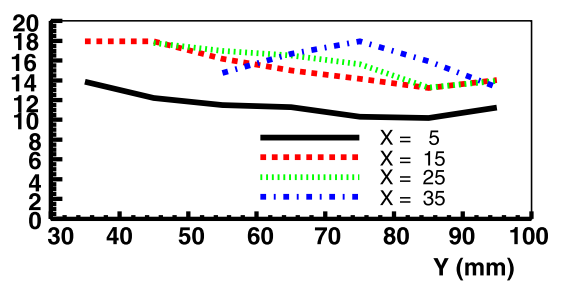

Mean signal in channel 3

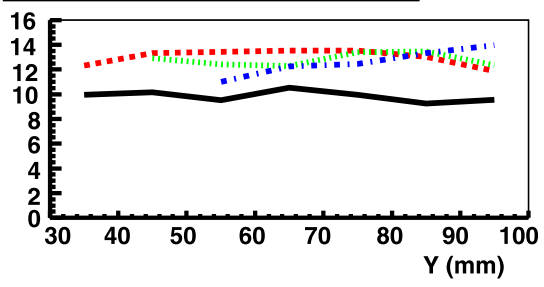

Mean signal in channel 5

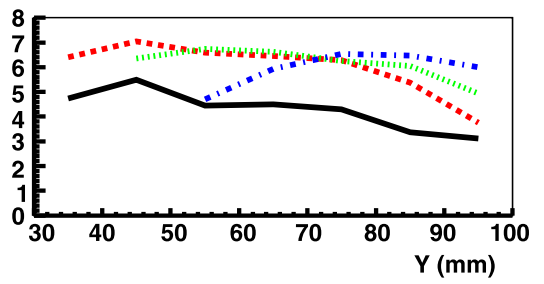

Mean signal

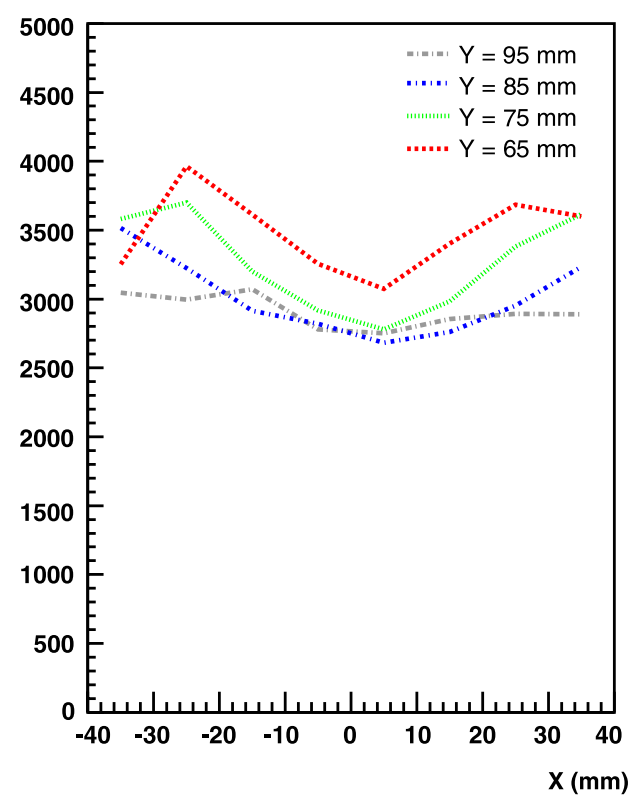

Mean signal in channel 2

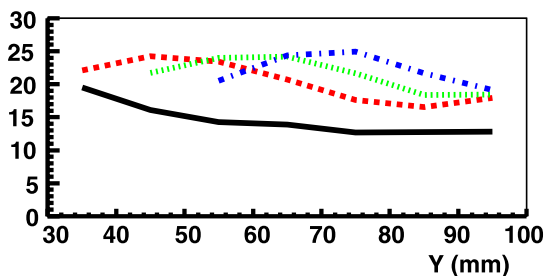

Mean signal in channel 4

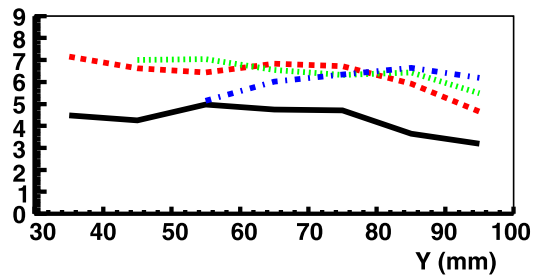

Mean signal in channel 6

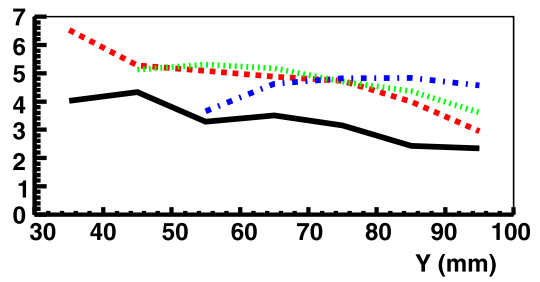

Mean signal

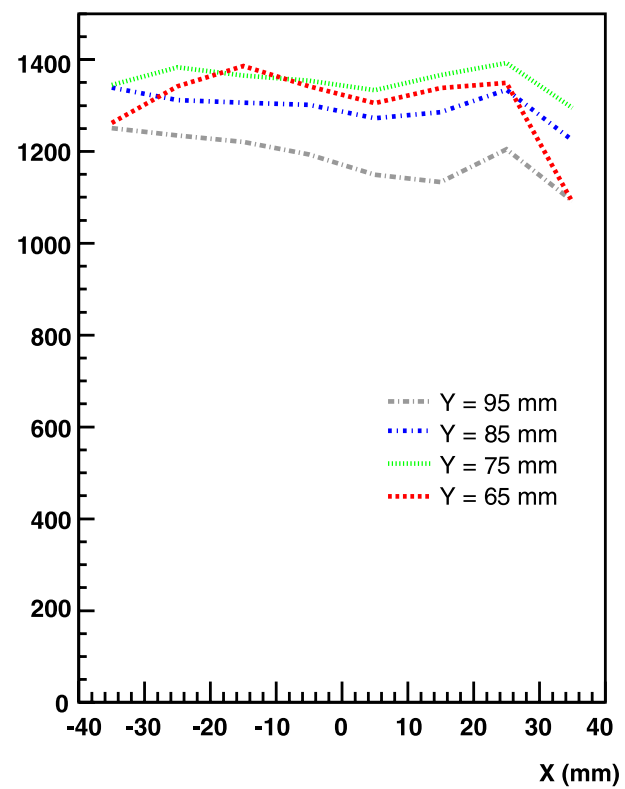

lower energies. The measured stochastic term of the energy resolution was found to be $187 \%$. The constant term is around $18 \%$. For energies above $100 \mathrm{GeV}$, the resolution is found to be below $25 \%$.
4. The response as a function of the position on the front face of the calorimeter shows non uniform variation, more enhanced in the electromagnetic section of the prototype. Later changes in the design of the calorimeter, be- 
Fig. 27 A scan over $x$ at $y=75 \pm 5 \mathrm{~mm}$ for electrons (left) and pions (right) of $80 \mathrm{GeV}$ energy
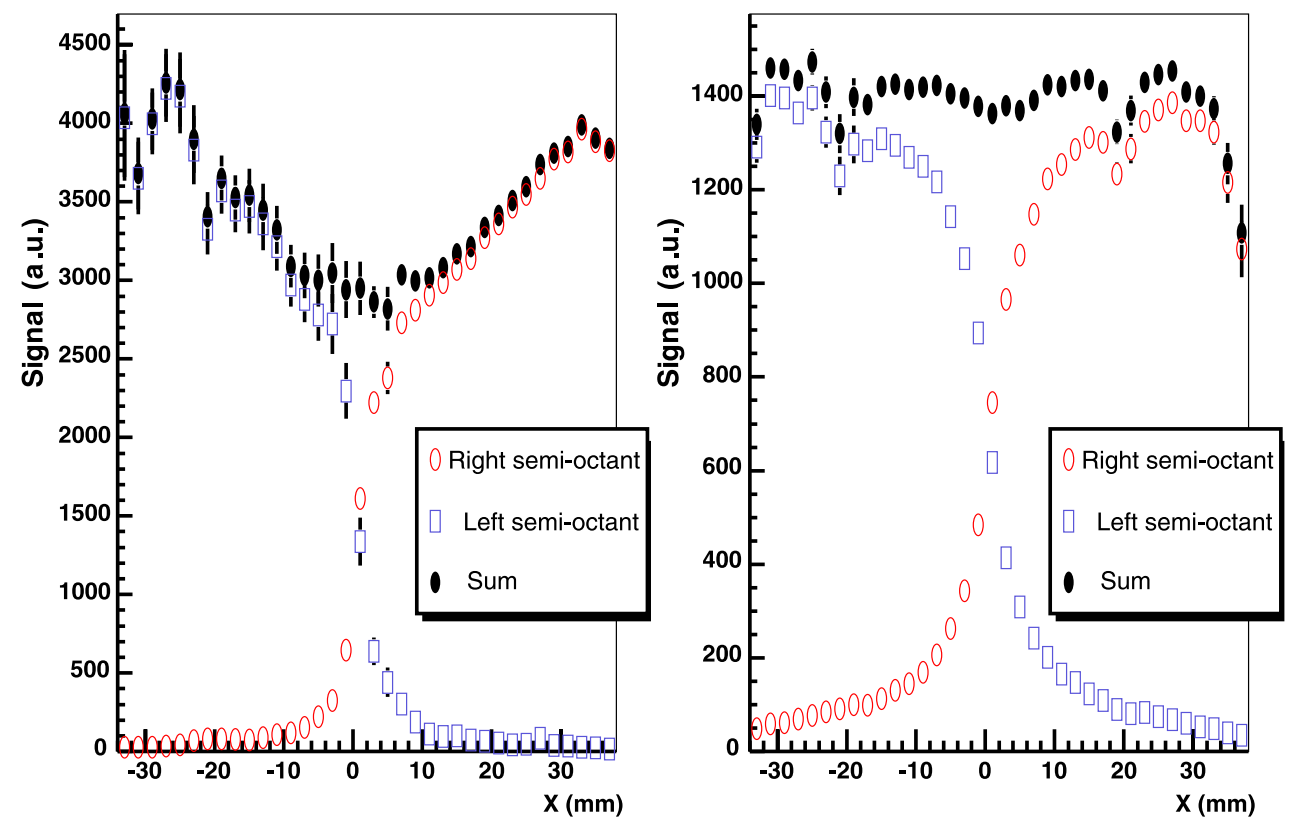

Fig. 28 Differential distribution over $x$ at $y=75 \pm 5 \mathrm{~mm}$ for the electromagnetic (left) and hadronic (right) showers of $80 \mathrm{GeV}$ energy

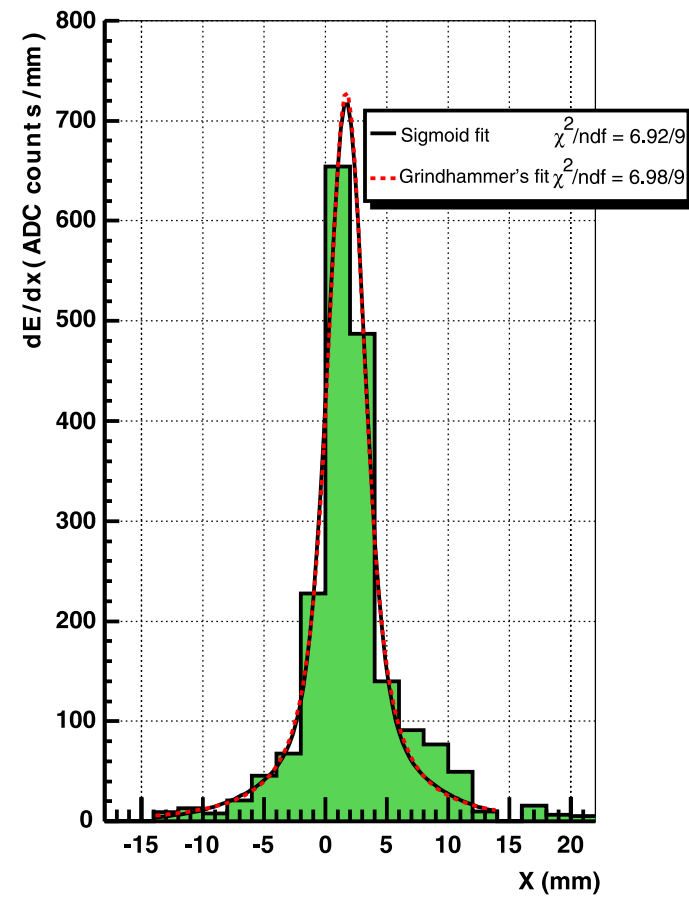

lieved to contribute towards improving the uniformity of the response, resulted in a new prototype that was beam tested in 2009.

5. The energy resolution of the calorimeter is sufficient for both the p-p and heavy-ion research programs planned.

Acknowledgements The authors wish to thank Dragoslav Lazic and the CMS HCAL group for their valuable technical support and excel- lent collaboration at the $\mathrm{H} 2$ beamline at CERN, the SPS Coordinator Christoph Rembser and the SPS accelerator staff for delivering a good and stable beam, as well as Fedor Guber (INR) and Evgueni Vlassov (ITEP). This work is supported in part by “A. G. Leventis” Foundation (Hellas), Hellenic GSRT under programme EPAN and by the Russian Foundation for Basic Research grant 08-02-0615. D. d'Enterria is supported by the 6th EU Framework Programme (contract MEIF-CT2005-025073). 
Fig. 29 Integral planar containment for the electromagnetic (left) and hadronic (right) showers of $80 \mathrm{GeV}$ energy
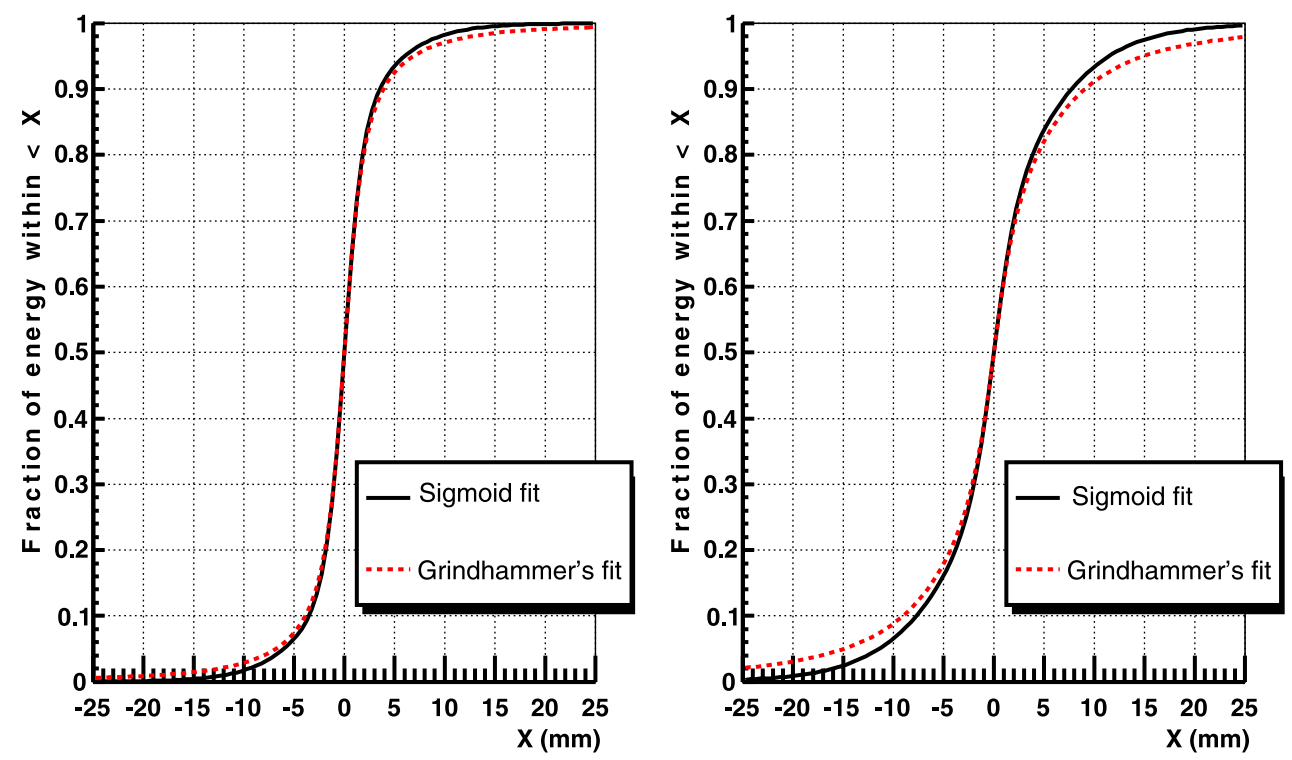

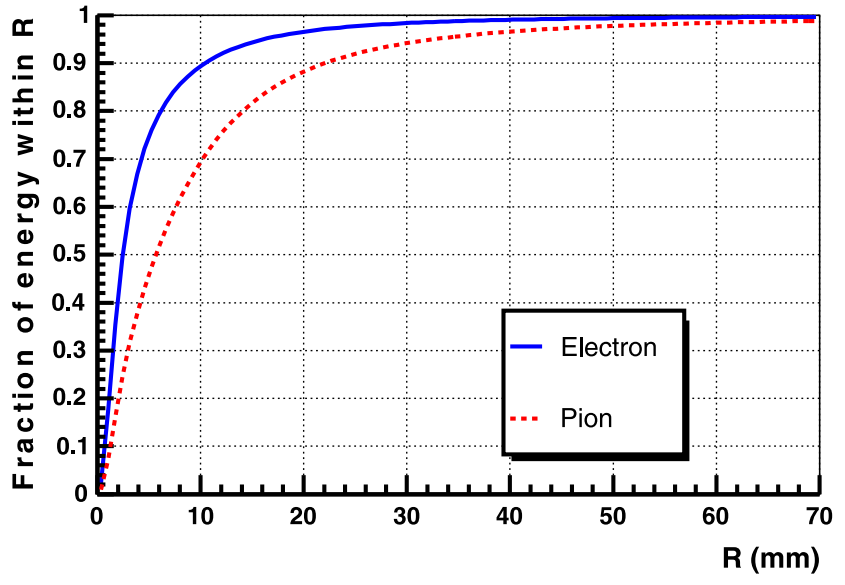

Fig. 30 Radial containment the electromagnetic (left) and hadronic (right) showers of $80 \mathrm{GeV}$ energy

Open Access This article is distributed under the terms of the Creative Commons Attribution Noncommercial License which permits any noncommercial use, distribution, and reproduction in any medium, provided the original author(s) and source are credited.

\section{References}

1. CMS-CASTOR group, CASTOR Engineering Design Report: A Calorimeter for CMS at Very Forward Rapidity (CMS, 2007)

2. M. Albrow et al. (CMS/TOTEM Collaboration), CERN-CMSNOTE-2007-002, 2007

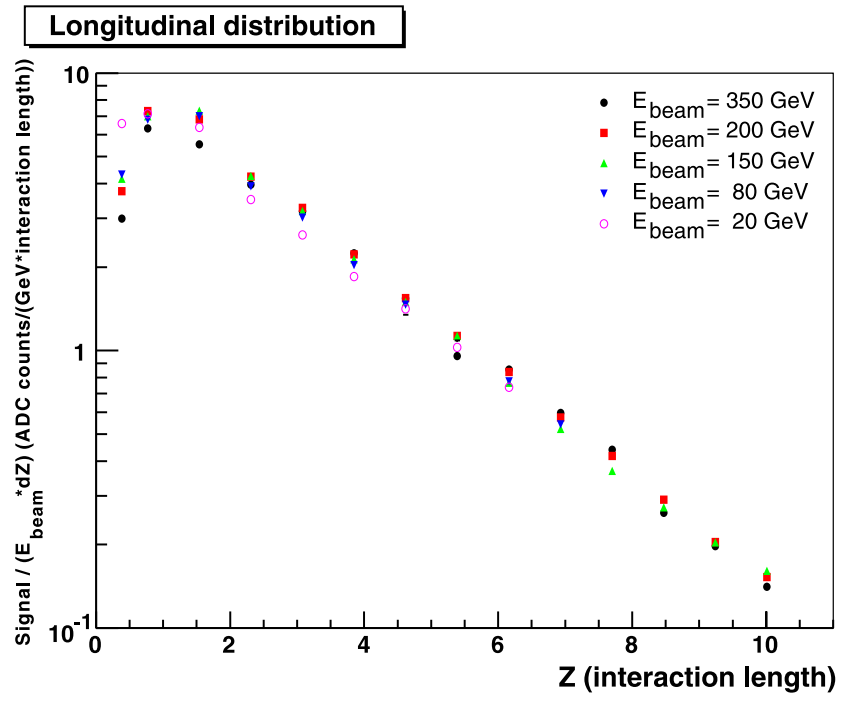

Fig. 31 Longitudinal profile for pions of different energies. The mean response of the calorimeter was normalized to the beam energies

3. D. d'Enterria (ed.) (CMS Collaboration), J. Phys. G 34, 2307 (2007)

4. X. Aslanoglou et al., Acta Phys. Pol. 39(6), 1429-1453 (2008)

5. X. Aslanoglou et al., Eur. Phys. J. C 52, 495 (2007)

6. T. Zimmerman, J.R. Hoff, IEEE J. Solid-State Circuits 39(6), 895905 (2004)

7. G. Grindhammer, S. Peters, hep-ex/0001020 